\title{
Failure analysis of performance vehicle mechanical gear
}

\author{
Antonios D. Tsolakis, Konstantinos I. Giannakopoulos ${ }^{*}$ and Andreas S. Gkertsos \\ Department of Mechanical Engineering, Piraeus University of Applied Sciences, Greece
}

\begin{abstract}
Gears are the main machine element in vehicle power transmission trains. In commercial vehicles, power transmission trains design complexity consists of the determination of the loading parameters which depends upon the working environment of the performance vehicle. In the present work a gear failure under dynamic loading is investigated. The failed gear is part of a 2 speed gearbox of a Rotax DD2 2-stroke OpenWheel performance vehicle (go-cart type). The gearbox was in racing conditions when failure occurred. The vehicle was speeding in 2 nd Gear at wide open throttle when instantaneously the driver by fault downshifted to 1 st gear. The result was for the first gears break a whole piece and eventually the system to go out of operation. Chemical composition of the examined metallic components was determined via EDAX chemical analysis.
\end{abstract}

\section{Introduction}

Gear is one of the most fundamental structural component when it comes to motion or power transmission. In order to transmit power or motion, from one specific point in a mechanical system, to another one, a number of gears can be used in such configuration that the desired output is achieved. Based on the power needed in the output of a mechanical system, multiple gears are used in order to multiply or reduce the specific characteristics of input element (torque, RPM etc.). Transmission units are mechanical systems, composed by multiple gears, that provide a torque increase and RPM decrease in order to carry out tasks that require more torque that the output of the motor used. Depending on the power requirements of the application, the mechanical properties of the gears are calculated in order to have sufficient strength to carry out its task, while, on the same time, minimizing the weight and production cost of the element. While gears are engineered to withstand the loads of each application, a number of failures modes can take place that will put the whole system out of service. Fatigue failure [1-4] is one of the most common failure modes. It occurs when a gear is subjected to dynamic loads that do not exceed the maximum allowed force that the component can handle. By altering the load, the mechanical structure gets weaker and eventually fails to support the load any further.

As Goksel Saracoglu and Ahmet Yapici state [1], the girth gear that they investigated in their analysis, did not exceed the maximum allowed load for the gear teeth to fracture. Instead, the support of the ring gear that was insufficient, lead to a cyclic condition of loading and unloading the gear teeth, that caused fatigue failure.

\footnotetext{
*Corresponding author: kgiannakopoulos@ teipir.gr
} 
Gang Shen [2], explored the causes of failure of a wind turbine planetary gearbox that went out of service, few years after the manufacturing of the wind turbine. This is far below the engineered lifetime of such constructions. After inspecting the fractured gears of the turbine gearbox, hardness measures were performed both on the gear surfaces as well as the bearing surfaces that were fit together by interference fit. Surface hardness and tolerances were all according to the engineering specifications so the operating conditions were taken into further analysis. Due to the working environment of wind turbines (unpredicted wind conditions, humidity, excessive temperature ranges), the loading of the gearbox is unstable that causes the whole system to suffer fatigue. The internal tolerances of the gear tooth profile in addition to the mounting tolerances, lead to uneven load transfer to the teeth of the gear that initiated fatigue sources. These were formed in terms of surface cracks. By extending the working time in these conditions, the surface crack propagated in the core of the gear and finally caused fracture.

Khaldoon F. Brethee [5], demonstrates a thorough analysis about gear wear, by using a gearbox composed by helical gears. In his paper, a state of the art mechanical system is used in order to produce all possible scenarios that take place in a gear component at any possible case. The gears were subjected to variable loads at multiple operating speeds, with vibrations that simulate the effects of the supporting bearings, motors etc. The gearbox was gradually guided to fail due to wear, and measurements were taken at specific time frames, that were later compared to the numerical outputs. In this way, the propagation of wear was studied and the effects of it at various time points was noticed. Using this analysis, gear wear prediction methods can be designed in order to minimize the failure possibility.

In addition to all failure modes mentioned above, gear pitting [6] and scoring sum up the category of the biggest causes of gear failure.

In this paper, a gear failure analysis is performed in a straight-tooth gear from a high performance engine gearbox. The gear under investigation is part from a 2 speed gearbox from a Rotax DD2 racing go-kart. The power flow can be view on figure 1 . The power from the crankshaft (1, Fig.1) is transferred to a centrifugal clutch drum (4, 9, Fig.1). At idle RPM the drum is not engaged so the power transmission is interrupted at that point. When engaged, the power is transferred from the clutch drum to the pinion shaft $(20$, Fig.1). A 1 st stage reduction is done at this point from mating gears 9-18 (see Fig. 1). The pinion shaft (16, see Fig.2) is engaged with the wheel shaft (5, see Fig.2) by a 2 speed gearbox. The gears 6 and 7 are mated to the pinion shaft and provide the 2 gear ratios. By selecting 1 st or 2 nd gear, the driver moves the clutch (11, Fig.2) to engage with the gears. The clutch pins are inserted instantly into the grooves of the gear and the mating is done by impact.

While the engine was on operation (race condition) at wide open throttle (appx. 12500RPM), the race driver, accidentally down-shifted from 2 nd gear to 1 st. Due to the fact that the circuit was at racing conditions (a lot of rubber disposal was on the track which caused it to be "sticky"), the rear wheels could not slip in order for the torque to be transferred to the wheels, so the power transmission stopped at gear 6 (see Fig.2), eventually causing fracture. The facture caused was not at the root of any tooth but it ripped a whole part of the gear with several teeth on it. 


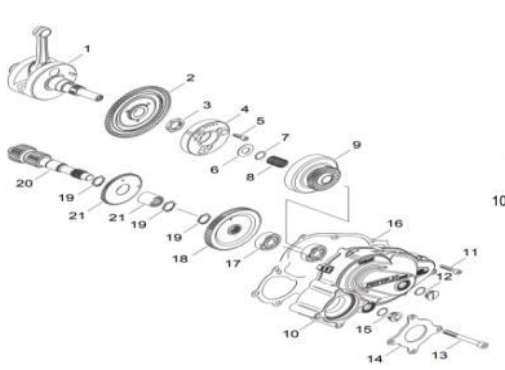

Fig. 1 Centrifugal clutch.

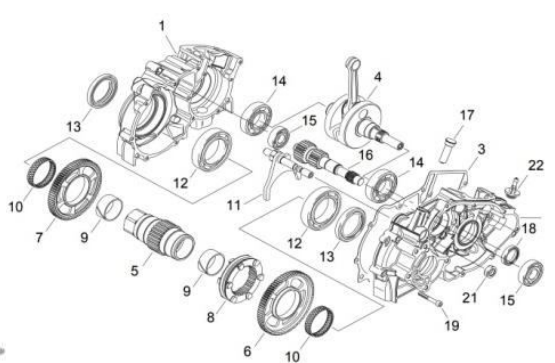

Fig. 2 Crankcase components.

\section{Methods}

\subsection{Chemical analysis}

For the determination of the material, chemical analysis was done to the fractured piece of the gear via EDAX chemical analysis. 3 samples were taken in order to validate the results, and below is an average value of each element. Fig.4a, $4 \mathrm{~b}$ and $4 \mathrm{c}$ show each chemical analysis independently.

$\bullet 93.05 \% \mathrm{Fe}$

$\bullet 3.02 \% \mathrm{Cr}$

$\bullet 3.04 \% \mathrm{Mn}$

$\bullet 0.88 \% \mathrm{Si}$

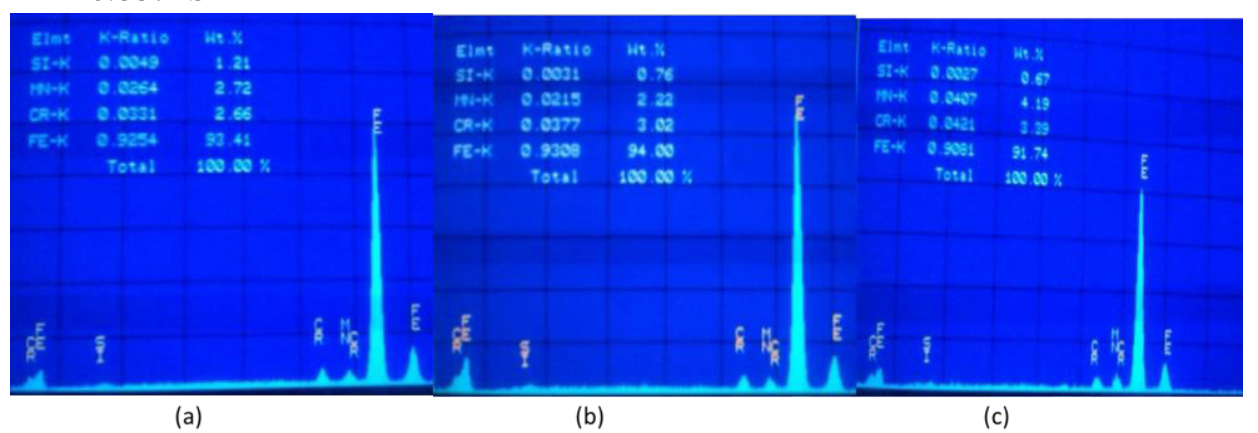

Fig. 4 Different chemical analysis results depicted. $4 \mathrm{a}$ on the left edge and $4 \mathrm{~b}$ on the middle and $4 \mathrm{c}$ on the right edge of the gear .

\subsection{D Parametric Modeling}

The 1st stage of the failure analysis consists of the creating a mathematical 3D model of the fractured gear through using SolidWorks ${ }^{\mathrm{TM}}$ 3D modeling software. The gear is a 77 teeth straight gear with $\mathrm{m}=1.5$. The gear tooth profile was modeled using equation driven splines to create the involute curve. The equations used can be seen below, where A refers to the radius of the tooth base circle. (Fig. 5a)

$$
\begin{aligned}
& x(t)=A(\cos (t)+t \cdot \sin (t)) \\
& y(t)=A(\sin (t)-t \cdot \cos (t))
\end{aligned}
$$

The $3 \mathrm{D}$ mathematical model is created using solid modeling techniques. In Fig.5b the complete model can be seen. 

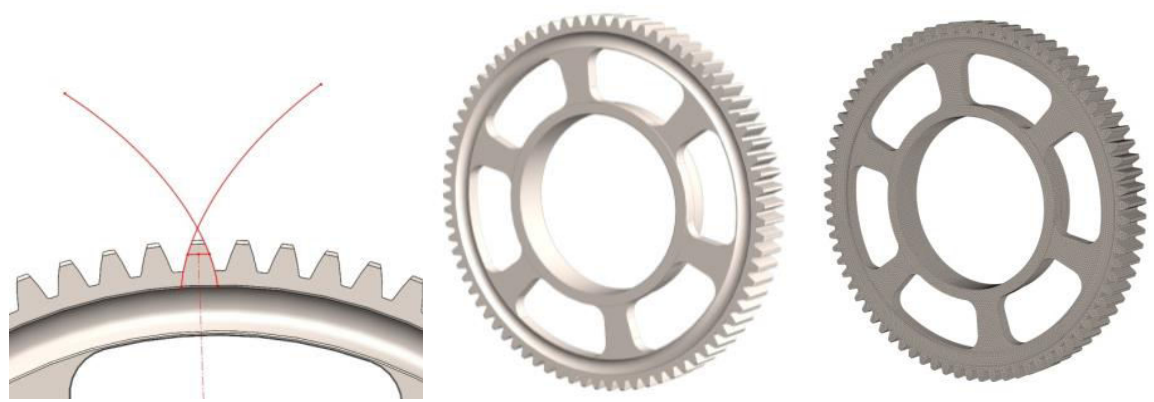

Fig. 5 (a) tooth profile equation driven curves, (b) 3D Mathematical model of the gear under investigation. (c) Mesh model of the gear.

\subsection{D Model meshing}

The process begins by subdividing the model into small pieces of simple shapes (elements) connected at common points (nodes). The software estimates a global element size for the model taking into consideration its volume, surface area, and other geometric details. The size of the generated mesh (number of nodes and elements) depends on the geometry and dimensions of the model, element size, mesh tolerance, mesh control, and contact specifications. For a more accurate solution, a smaller element size may be required.

Meshing generates 3D tetrahedral solid elements, 2D triangular shell elements, and 1D beam elements. A mesh consists of one type of elements unless the mixed mesh type is specified. Solid elements are naturally suitable for bulky models and they are used in this analysis. The type of mesh used to mesh the 3D model is parabolic tetrahedral which is defined by four corners, six mid-sized nodes and six edges. The parameters of meshing can be seen in Fig.6B.

\subsection{Load calculations}

At the time of failure the go kart was speeding in 2nd gear at wide open throttle. From the engine performance graph [8] and maximum output power for a DD2 engine is $24 \mathrm{~kW}$ at 12,500RPM. Converting the output power of the engine into torque, the maximum torque occurs at $10,500 \mathrm{RPM}$ and $22 \mathrm{~kW}$ of power which leads to $20 \mathrm{Nm}$ of torque. For calculating the maximum torque, the following relations was used:

$$
\text { Torque }(\mathrm{Nm})=9.5488 \times \operatorname{Power}(\mathrm{kW}) / \operatorname{Speed}(\mathrm{RPM})
$$

Having calculated the maximum torque, a conversion to applied force must be done. The second gear consists of 62 teeth and is engaged with a pinion of 35 teeth by default. Many gear ratios are used in each race for maximizing the performance of the engine in each circuit. It this occasion the gear ratio is $1 / 1.77$. A conversion of the gear revolutions is done in first stage,

$$
\text { PinionRPM }=\text { ClutchRPM } / \text { 1,77 = } 10500 / 1,77=\text { 5932RPM }
$$

and the same is done for torque,

$$
\text { PinionTorque }=\text { ClutchTorque } * 1,77=20 * 1,77=35.4 \mathrm{Nm}
$$


The maximum torque of the pinion is calculated and so the maximum force will be calculated next. The 1 st gear ratio is $24 / 77$ or $1 / 3.2$. The pinion torque at the moment of failure is $35.4 \mathrm{Nm}$. The maximum force applied at that moment is calculated through the following equations:

$$
\begin{gathered}
\mathrm{F}_{\mathrm{n} 1}=\left(2000 * \mathrm{~T}_{1}\right) /\left(\mathrm{d}_{1} * \cos (\alpha)\right) \\
\mathrm{F}_{\mathrm{n} 1}=(2000 * 35.4) /\left(36 * \sigma v v 20^{\circ}\right)=70800 / 33.8289=2093 \mathrm{~N}
\end{gathered}
$$

where $T_{1}$ is the maximum torque in $\mathrm{Nm}$ and $\mathrm{d}_{1}$ is the pinion base diameter in $\mathrm{mm}$. From the above calculations, the resulting maximum force applied on the gear tooth is 2093Nt. The previous parameters can be seen in Fig.6

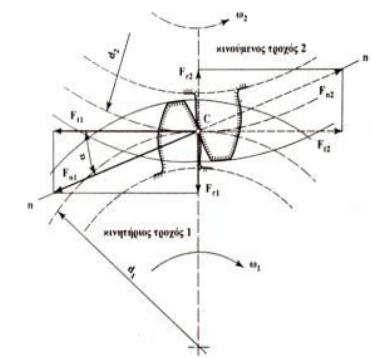

Fig. 6 Tooth load application detail.

\subsection{Simulation case studies.}

Having completed the parameters of the analysis to begin, multiple case studies must be run in order to verify which tooth was engaged at the moment of failure. For this reason 3 case studies were selected. In the first one, the first tooth of the fractured piece is assumed to be engaged. In the second one, a middle tooth is assumed to be engaged and in the last one, the last tooth of the fractured piece. For all three scenarios, simulation is run independently.

The results of the max von Misses stress are shown in the Figures 7a, 7b, 7c for each scenario respectively.

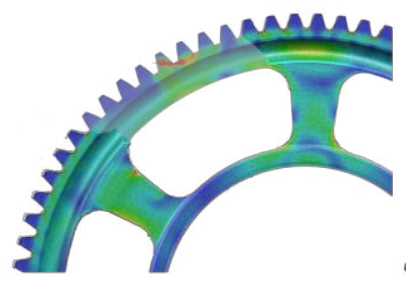

(a)

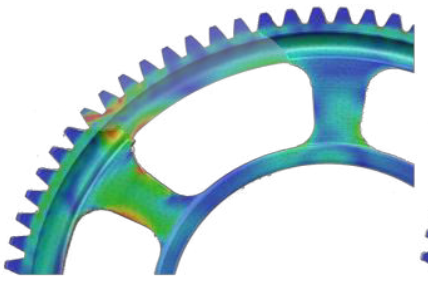

(b)

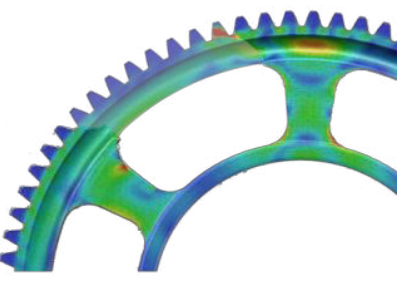

(c)

Fig. 7 Multiple Simulation scenarios can be seen. (a) first tooth engagement during fracture, (b) middle tooth engagement, (c) last tooth engagement.

Comparing the results, it is clear that the first case study is valid as the stresses developed in the gear are at the points that the gear suffered fracture. There is a special point in which the simulation presented a concentration of stresses in the inner part of the gear (below the outer surface). That point, is the point where the fractured changed direction and a corner was formed. See Fig.7a 
Following the Von Misses stress calculation, the 1st Principle stress is calculated and the results are listed in Figures 16, 17 and 18. The case studies were simulated for a given element size of the mesh. For this reason, the results cannot be validated unless multiple simulations are run for different sizes of elements during meshing. This process is called results convergence. Unless all stresses converge after a specific size of element, the simulation cannot be validated. The convergence graph for the 1st case study, max Von Misses stress as well as 1st Principle stress can be seen at Fig.8a and Fig.8b The above mentioned graphs were extracted for element sizes of $2,1.5,1.25,1,0.75 \mathrm{~mm}$.

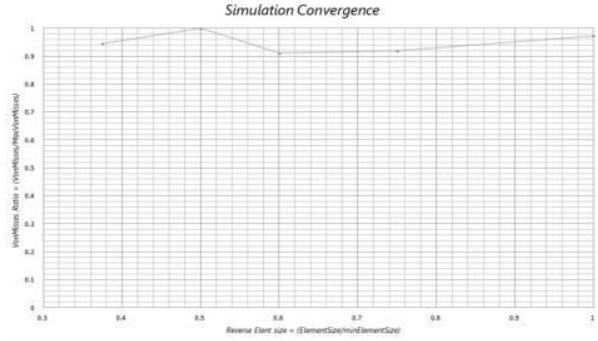

(a)

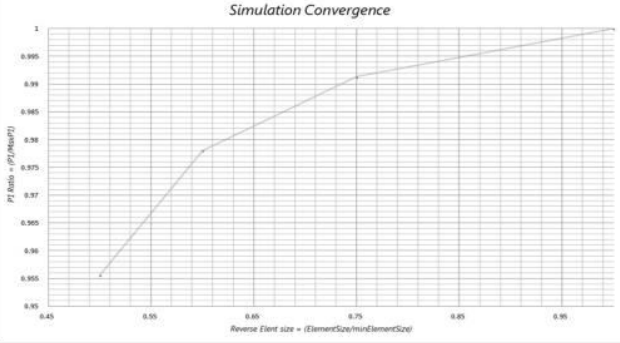

(b)

Fig. 8 (a) Convergence graph of max Von misses stress and (b) convergence graph of 1st Principle stress.

\section{Results}

The gearbox was in racing conditions when failure took part. The Go-Kart was speeding in 2nd Gear at wide open throttle when suddenly the driver accidentally downshifted to 1 st gear. The result was for the first gear to break a whole piece and eventually the system to go out of operation. In figure 9a,b, the fractured gear and the FEA solution of the fractured gear are shown.
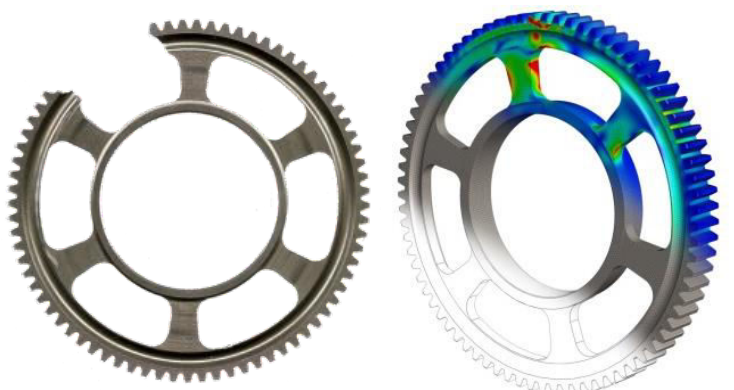

Fig.9 (a) The gear after suffering fracture, (b) the FEA solution superimposed on the gear model

Thereinafter of what were studied by the method of finite element analysis, the specimen and specifically their fractured surfaces were tested with the scanning electron microscope (SEM). The surfaces which were studied for their fracture mechanics behavior are the points where the finite element analysis qualitatively showed signs of severe mechanical stress. As emerged above, the maximum Von Misses stress (637MPa) seems that it does not exceed the maximum UTS strength $(1100 \mathrm{MPa})$ of the material which is consistent to the fact the breaking did not occur from one dimensional loading but it is likely due to repeated loading phenomena (fatigue), findings of which will be explored in this metallographic investigation.

This observation of the finite element analysis can be assigned in addition to material fatigue or in shock load which may be the cause of breakage of the material due to the rapid 
development of the internal stresses at the impact of the material, resulting in the accumulation of disorders within the slip planes of the material and the instantaneous stopping (due to congestion), or existence, either macroscopic or microscopic imperfections in the material structure, which potentially can be a starting point of breakage.

Fig.9a shows the fracture surface of the main part of the gear which exhibit ductile fracture phenomena in the central regions of the surface. In these areas, an intense terrain is present with characteristic crystal detachment areas, whereas in areas near the edge of the gear body mixed fractured phenomena is visible with intense brittle nature (areas of low roughness with evident the slit effect). The existence of such areas on the edge of the main part of the gear may be linked to a surface heat treatment process such as hardening, which explains the presence of different type of fracture in relation to the main body of the gear. Figures $9 b$ and $9 c$ are enlargements of the surface area that was studied in fig.9a. In these figures, it is apparent the phenomenon of successive crushing levels on the edge of the specimen, especially in the mixed fractured area with limited relief. From these two figures, it is possible that the material failure mechanism can be attributed to a fatigue phenomenon of the above layers of the material near the outer surface, to which may be contributed by the hardening heat treatment of the material with a final result, the accumulation of stresses in areas near the edge of the material from which began the phenomenon of fracture. The characteristic "waves" of fatigue are visible with characteristic concentric development, fact that shows that the fracture point of the material may be in the area showed in Fig.9d.

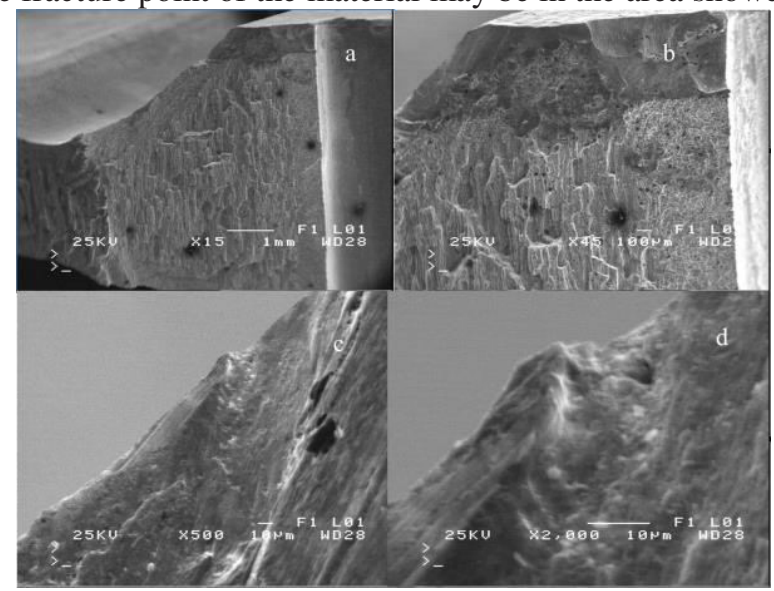

Fig. 9 Fractured surfaces observed by SEM microscope. Different magnifications are presented (a) $\mathrm{x} 15$, (b) $\mathrm{x} 45$, (c) $\mathrm{x} 500$, (d) $\mathrm{x} 2000$.

\section{Conclusion}

Observing the results of the finite element analysis, it is evident that the point where the gear was fractured, there is stress concentration. Stresses that are not sufficient to cause fracture under one dimensional loading because the maximum developed stress does not exceed the UTS strength of the material. At this point, there are qualitative results the justify the point where the failure occurred.

The next step was the study of the fractured surface of the gear. Each failure leaves some "traces" to the point where it is taking place (in this case, the surface created by the break). The aforementioned study was done by scanning electron microscope SEM.

Watching the photos exported from the microscope, there are two distinct areas. The surface area of the main body of the gear which shows traces of ductile fracture, and the area on the surface of the gear which exhibits strong brittle character. This variation is 
because the gear was subjected to surface heat treatment and the crystal structure had changed in the outer surface in relation to the main body.

Successive levels of fracture in the main body of the tooth, indicate the the failure resulted from fatigue of the surface layers of the gear (which had been heat treated), and then transmitted to the interior, resulting in complete breakage of the portion.

\section{References}

1. Goksel Saracoglu, Ahmet Yapici, EFA, 55, 139-147 (2015)

2. Gang Shen, DongXiang, Kan Zhu, Li Jiang, Yinhua Shen, Yanlin Li, EFA, 87, 96-110 (2018)

3. Thomas J.Mackin, Nash Anderson, Silvia Aguilar, Griffin Beemiller, Bert Copsey, DustinDraper, ErikEckberg, Leah Herbert, Daniel Layton, EFA, 32, 334-347 (2013)

4. Xiaofeng Qin, Ruiqiang Pang, Xingguo Zhao, Feng Li, EFA, 84, 70-76 (2018)

5. Khaldoon F. Brethee, Dong Zhen, Fengshou Gu, Andrew D. Ball, Mechan. And Mach. Theory, 117, 210-229 (2017),

6. J. Kattelus, J. Miettinen, A. Lehtovaara, Trib. Int., 118, 458-464 (2018) 